\title{
Methodological Principles of Formation and Development of Entertaining Institutions
}

\begin{abstract}
The generalization of the typological, functional, and architectural organization of entertaining establishments will contribute to the further improvement of their development in modern conditions. The purpose of the article is to identify the systemstructural principles of the architectural environment typology of entertaining establishments and to consider the features of the architectural and spatial structure of leisure objects in accordance with the hierarchical levels of their environment's formation and development.

The research was based on A systematic approach that allows us to consider the architectural environment of entertaining institutions as hierarchically subordinate to the integrity. Methods of historical, comparative and typological analysis were also used.

The article summarizes the features of the entertaining institutions' typology. The specifics of their location in the architectural environment are considered. Features and examples of their functional and spatial solutions, including existing buildings and structures, are considered, taking into account the transformation and adaptation of them for entertaining establishments. The hierarchical levels of formation and development of the architectural environment of leisure objects are revealed.
\end{abstract}

Key words: entertaining institutions, typology of the architectural environment of leisure, system-structural principles, hierarchical levels, design

\section{Introduction}

Objective patterns of entertainment establishments' formation and development and various means of influence of designers on their creation can not be considered outside the context of socio-economic, economic, typological, technical, aesthetic and other aspects and factors. Each of them, in one way or another, affects both the conceptual solution and the final realization of leisure facilities. The key role in the formation of their environment belongs to typological factors, which include features of the typology of public buildings, the improvement and development of their functional-spatial structure, elements of improvement and arrangement [1].

In recent decades, A large number of shopping and entertainment centers has being built. Such facilities include several basic functions - shopping, entertainment, food, which is extremely convenient and attractive to the public. People can come to them themselves, in the circle of friends or family, on matters or to rest. Such multifunctional institutions are part of the public space of the city, which at the same time has certain limits and is usually located in A separate volume. At the same time, insufficient study of the problems of entertaining leisure facilities' functional and architecturally-spatial decisions predetermine the need to consider the current trends in the organization of their typological and planning structure, various architectural and compositional solutions.

The research of this subject is present in the works of various scientists and specialists $[2,3,4,5]$, which are devoted to the analysis of the processes of formation of the leisure and entertainment industry in general and entertainment areas in particular. At the same time it is expedient to generalize the principles of classifications and the corresponding design decisions of entertaining institutions. The generalization of typological and architectural-spatial organization of entertainment institutions will contribute to the further improvement of their development in modern conditions.

The purpose of the article is to identify the features of the typology and the formation of $A$ harmonious architectural environment of entertainment centers and the specifics of their compositional and spatial organization, in particular in terms of existing facilities' adaptation.

\section{Typological principles}

With the advent of new cultural models, life styles and leisure practices, the typology of entertainment establishments is expanding accordingly.

The most common is the classification of cultural and leisure establishments for:

- quantitative composition (from A small number of people to A number of international ones movements):

- the direction of activity (from the internal work of the institution to the impact on the environment through educational, recreational, recreational, vocational, social and cultural activities);

- demographic characteristic (rural, town, regional institutions of culture and leisure to the city and state); 
functional feature (from monofunctional and specialized to multifunctional institutions)

sexual characteristics (homogeneous and heterogeneous associations :

- social orientations (student, women, organizations for children and adolescents, clubs for military teachers, etc.), content of activities (sports, musical, religious, literary associations);

the age of leisure activities (youth, adolescents, the elderly) [4]

Sometimes the criteria for determining the typology are the goals and methods of organizing leisure activities (for example, A circle of beading, A club of chess players) or the structure of an entertaining institution (such as A water park, wellness center or an arboretum), which According to the German scientists $G$. Triesch and W. Ackenfels [5] it is expedient to consider the complexes Of culture and leisure, classifying them on: Ocken (institutions) aimed at th

at the creation (palaces and taining recreational or sports work (cultural and sports complexes, recreation centers, entertaining and recreation centers);

- establishments for which social work is the main (for example, with «heavy" teenagers, invalids), professiona orientation, provision of social-cultural assistance to the elderly, immigrants, women (social and cultural centers, social clubs).

Among the entertainment facilities, children's and family entertainment complexes are the most common. They are located in separate premises (stationary or temporary) or function as A structural component of another institution for example, shopping and entertainment center or museum). Entertaining centers in the system of shopping areas form the second group of entertaining complexes (ill. 1). The entertainment component is inherent in most com-
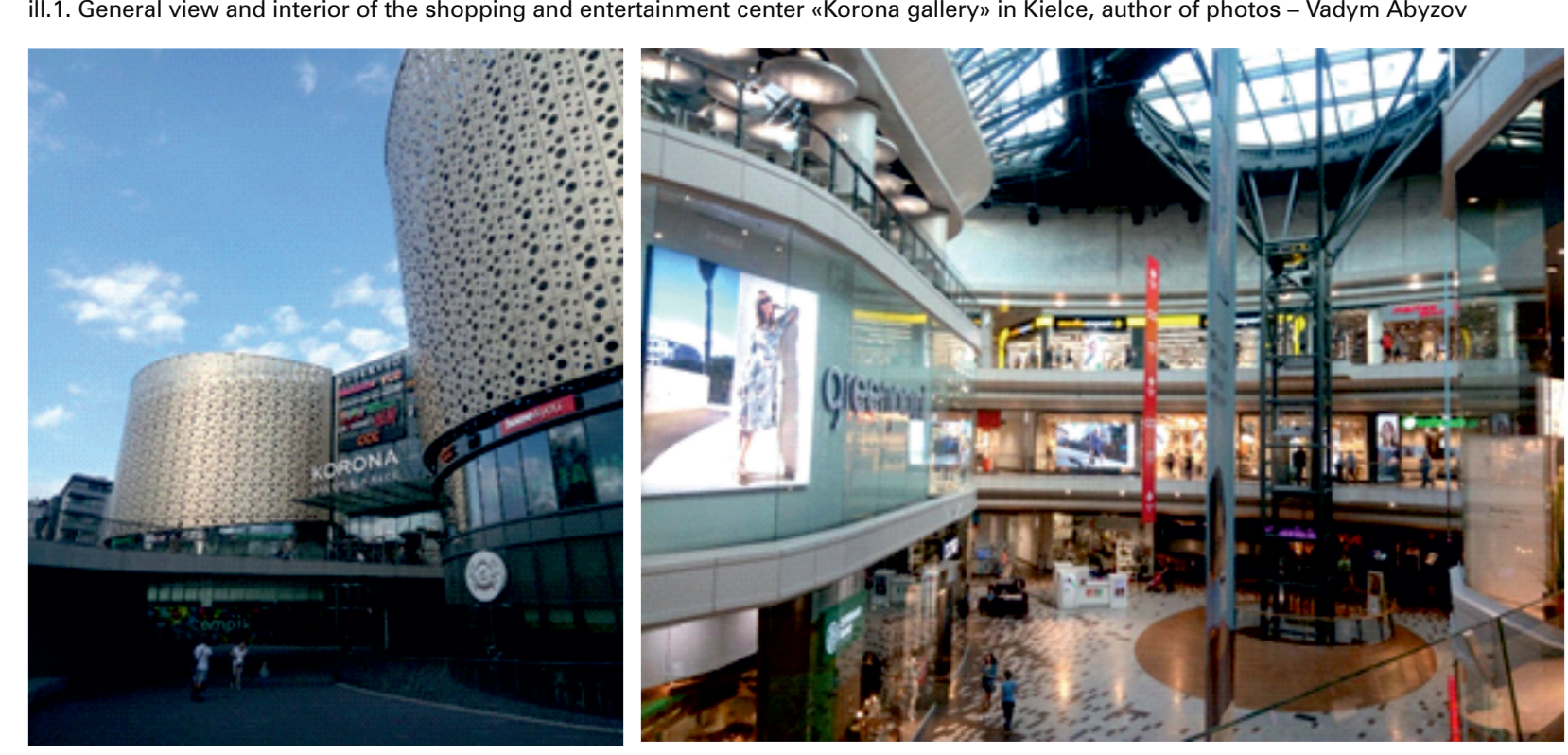

mercial objects of modern society, since it significanof the institution. The third group of entertainment complexes include amusement parks (parks of culture and recreation, theme parks, water parks). Recreational, entertainment, and sports and entertainment lons, fitness centers) form the fourth group of entertainment complexes. The fifth group includes entertainment and recreational complexes, the purpose of which is to organize an effective human leisure. Among the basic principles of service in entertaining and recreational complexes are the following: individual approach, complexity in the organization of leisure activities; systematic leisure choice and voluntary participation, theatricaliza,

The problems of the entertainment center are tho-

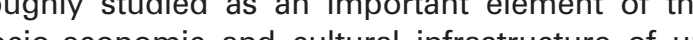
ban space, while the processes of modeling and formation of institutional structures, changes in individual motivations and benefits of the population attract many researchers' attention. In this connection should consider the typology of entertainment facilities for their urban location and territorial characteristics, namely: in cities, in rural areas It should be assumed that in the largest and large cities, tainment complex, there should be entertainment complexes of district significance in view of trans port and pedestrian accessibility. They are usually located at the intersection of main motor roads and hiking trails in the public centers of the city and the ping and entertainoper e functions of leisure can be located in close to facilities (paintball clubs, wellness centers, SPA-saconfiguring the city, ways of its optimization, transin addition to the central (main) city-wide enterdistrict and usually operate in the structure of shop-

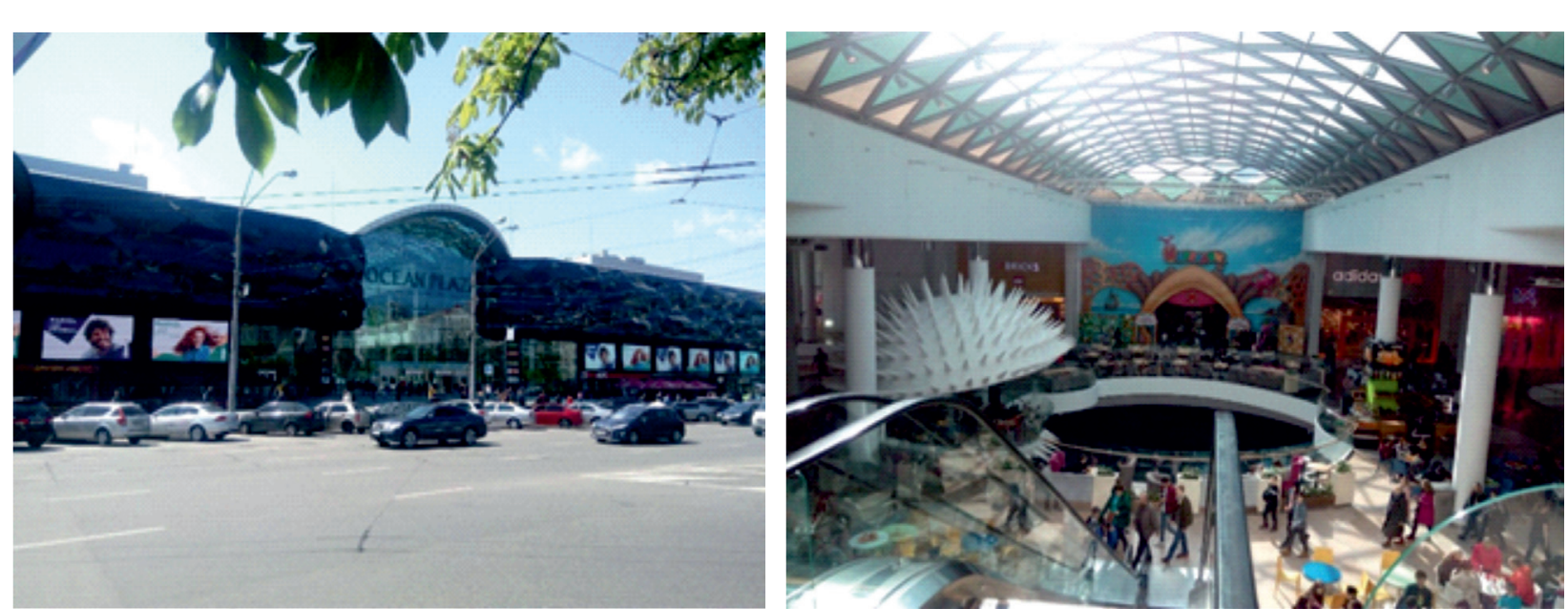

II. 2. General view and interior of the shopping and entertainment center uOcean Plazay in Kiev, author of photos-Vadym Abyzov

public housing and shopping centers of neighborhoods and large residential buildings. At the same time, for tourist and resort cities, the structure of entertainmen facilities should be more developed with the location in the recreational areas.

It is worth noting that in small rural settlements (up to 3-4 thousand people) the creation of A separate entertainment establishment will not always be effective and coseffective. Therefore, it is necessary to take into account the specifics of the settlements and to foresee the creation of inter-settlement entertainment complexes in large villages and small towns that could meet the needs of the population of small villages, taking into account their respective transport accessibility. In small settlements in this case it is expedient to equip separate functional components of culture and leisure which can be located the buildings of village councils, schools, clubs, etc. The modern entertaining complex is an idea of concentration of divere fors is the complexity of services provided A cinema area and A restaurant courtyard, which should unite the general concept Entertaining objects are rather complex and, at the same time, unique leisure establishments. Nu merous functions, concentrating in the activities of similar institutions, gurations. In addition the process of implementing these functions is carried out under the constant influence of various external factors, which must be taken into account when analyzing the activities of such institutions. To the considered typology's features we consider it appropriate to add another classification of entertainment facilities for placement in buildings, namely:

- in separate buildings, created for leisure:

-in the buildings of public and commercial complexes - in premises built-in to residential buildings:

in existing buildings and facilities with the conversion and adaptation of them for entertaining establishments. The first ones are located mainly in public centers of $\mathrm{ci}$ ties and recreation areas, the second ones are in entertainment complexes of the city and district significance. the third ones are in the centers of the microdistricts and large residential units. The last option for accommodating entertainment, which can be related to various urban development situations and territories, is becoming more widespread due to the large number of buildings that have lost their origina function and the economy of urban areas.

One of the interesting and useful examples of adaptation and renovation of buildings is the «Paladium shopping and entertainment center in Prague (the developer of "European Property Developmentw). On the site of the closed Capuchin monastery with St. Joseph's Church, barracks were built that were used until 1996. The small and modest church was preserved, and the barracks became the basis for the new building of the shopping center. Behind the From there, you can also enter the entertainment pital. The shops on all five floors are well in the cawith the most up to date traling equipmequipped mpressive windows and interiors, as works of art from which it is difficult to take $A$ look. This complex is $A$ good place not only for shopping, but also for leisure. More than three dozen restaurants, bars and cafes work to satisfy the taste of the guests. In the center of this object is an entertainment complex "Hrad Zabavy", which contains A children's zone Umka development studio, rides, slot machines for children of all ages, and clowns and animators conduct games and activities with children [6]. The complex «Paladium» became A vivid example of the poelements into the structure of the historic building. Another no less interesting example of the renovation of $A$ non-functional industrial building, namely the gas meters of 1896-1899, is the construction of A public building complex in the Gasometer City shopping center in Vienna (inc. T. Herrmann, F. Kapaun). The four former gas pipelines used to stooutdated, and in 1999-2001 they were re-profiled From th $A$ cozy courtyard with an unusual fountain. complex and have $A$ rest after outdoors shopping. pular architectural trend - the integration of modern re natural gas were closed, morally and physically 

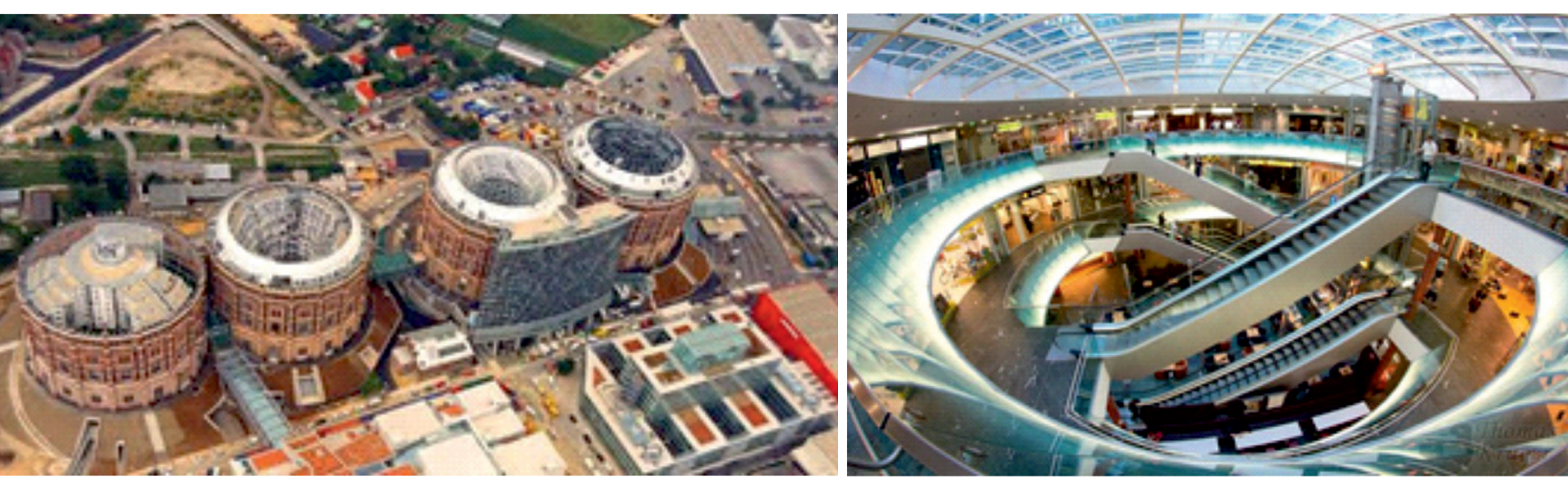

ill. 3. General view an

into the complex of Gasometer City TC. The complex of four gas storage facilities contains 615 modern apartment
apartments, offices, more than 70 shops, restaurants, A cinema, A conference room for 3500 people. The main and largest unit is located on 3 levels of the gas meter "A» and performs A trading function. The entire public shopping and entertainment complex is A 450-meter, 4-storey shopping center, located in each of the four gasometric buildings with the additional iEn center adjacent to them. In addition to the MMaccis $A$, the complex has restaurants, and in the center «Em is A cinema, bowling and billiards. In the basement of the gas meter "B» there is $\mathrm{A}$ concert hal «Die Bank Austria Gasometer Halle» for $1400 \mathrm{~m}^{2}$, accommodating 4200 people. The "Gazomether Hall» hosts huge concerts of pop, rock, jazz and even classical music, shows musicals, plays of modern opera, theater, cabaret, discos and night rave parties. The gas meters "C" and "D" are quite different among themselves, connected by A glass «bridge» extending from the main shopping center. At the bottom of the main mall is $A$ huge underground parking

The design of the intertiors of these entering of artistic design tools, the use of modern buidiy materials, exquisite color scheme and an effective and aesthetic lighting solution. Taking into account this useful interiors in the conditions of adaptation of premises of existing buildings and structures, the issues of adapting their spaces to the functions of leisure facilities taking into account modern forms and means of technical development should be comprehensively solved.

\section{System-structural principles}

In the system approach, the essence of which consists in the implementation of the requirements of the general theory of systems, the design object is considered as $A$ set of interrelated elements and components that form $A$ system that binds the components to $A$ common goal. Therefore, when forming leisure facilities, they should be divided into appropriate hierarchical levels of organization of the architectural environment, distributing them first to open and closed architectural systems. The first hierarchical level of open spaces of entertain- ment facilities will be urban structural unit, which determines the place and role of the object of leisure in the space of the settlement and its development taking into account the various urban planning and climatic conditions of the location, the characteristics of its natural and artificial environment. Moreover, this hierarchical level has the appropriate structural subleof the general layout of the city; the second - the level of urban development projects/structures and ensembles. The next hierarchical level of open spaces is the environment level of individual buildings and structures subordinated to the corresponding urban development and their environment as A whole. defines the principal functional and architectural-spathal organization of an entertainment facility. The las structural block will be the surrounding environment f the territorial fragments, such as various elements of the arrangement, entrance groups, small architecAs for the parking spaces, etc.

列 first hierarchical level of closed spaces is the orga nization of the entire internal environment of $A$ building or an ensemble of buildings and structures, which unites the complex with $A$ single internal spa ce. At this level, the overall design and composition che ment of the entire interior space, the conditions of ment of the entire interior space, the conditions of
its relationship and interaction with the open space of the environment are solved. The second hierarchical level will be the organization of the interna environment of specific leisure facilities of the building or complex, taking into account the connectio with other open and closed elements of the environment. At this level, as at the previous one, the conent, spatial composition, the overall color solution of individual functional components and premises are decided. The color scheme, lighting and selection of lighting equipment in the design of entertainWent facilties should also be given special attention. Well-chosen colors in conjunction with lighting equipment can significantly visually increase or decrease vels or subsystems, for example: the first - the leve chitectural environment of leisure institutions. The the room, create A comfortable and unique atmosphere for visitors and emotional effect.

The third structural unit is the subject content of the en vironment and the compositional and artistic solution the main system-forming elements and fragments of the interior of A particular room in the context of its genera environmental design. The elements of the subject conten of the architectural environment primarily include its equ pment, which has A functional purpose, such as furniture visual advertising, equipment, small architectural forms, elements of engineering infrastructure. Artistic design of equipment, along with the need for A comprehensive solution of specific functional, structural, ergonomic, economic conditions of the enving So the design of furniture flowe beds fountains, scultuTo to the general conceptual design. The saturation of the composition and the creation of the stylistic expressiveness of leisure objects is also due to the expressive deta ils of decor and the synthesis of arts. Therefore, the fourth hierarchical level will be an aesthetic solution of the details of decor and synthesis of arts. Harmonious synthesis of design objects with fine and applied arts (artistic ceramics, monumental and decorative painting, artistic textiles, sculptural compositions, consumer goods made of wood metal, glass, etc.) is A powerful means of their organic interaction and connection with the architectural environment Here one should also remember about the skillful use of the means of landscape art and phytodesign. After all, plants, herbs and flowers and their meaningful and aesthetic combinations are of great importance in the arrangement of the entertainment environment. Finally, the last firth level is the use of appropriate building materials and products. Skilful use of traditional building materials (stone, ceramic, wood) and their texture provides special natural artistic-figurative characteristics of

The high development of scientific and technologica progress and the introduction of nanotechnologies re veals broad aesthetic and artistic features, along with traditional, innovative products and materials (concrete and reinforced concrete, anodized metal, glass and other materials and products from mineral melts, poly-

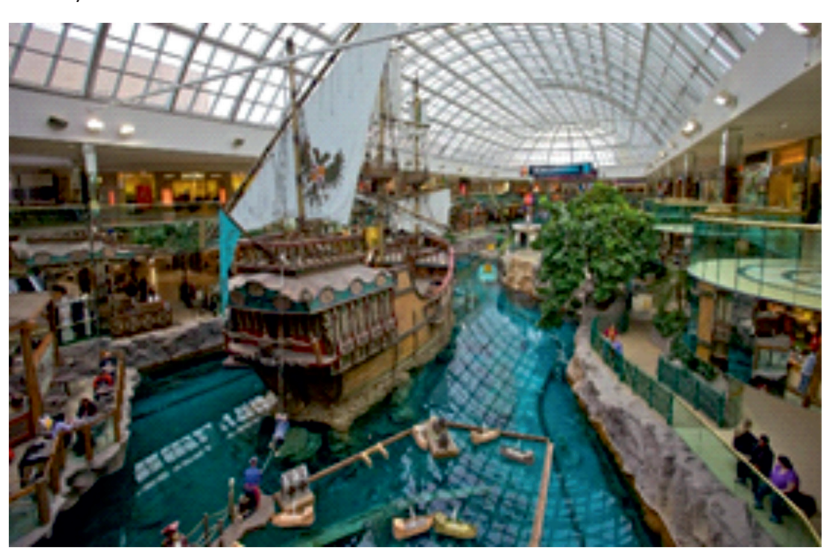

mer materials) for the creation of extraordinary compositions and implementation daring creative design ideas. It is worth adding to the above that it is necessary to take into account the fact hat the design of the entertainment complex must be periodically updated and should not be static. At he same time, it is important to ensure velictic integrity of the spatial and artistic-figuative solutions of the complex.

Conclusions

the article the features of formation of the architectural environment of entertaining establishments are investigated. The features of the typology an design of entertaining establshments are generaliand bulding are conidered. An analysis of the and buillins are considered. An analysis of the in conditions of adaptation of premises of existing buildings and structures indicates the economic expediency and perspective of such an approch and their re-engineering and renovation, taking into account the initial architectural solutions.

When forming entertainment centers it is necessary to solve complex problems of their rational functional spatial, constructive, technological, engineering orga nization. The design of the objects of the environment and their elements must be combined with A genera composition that attracts visitors with an attractive look and $\mathrm{A}$ high degree of comfort, and to provide the stylistic integrity of the spatial and artistic solution of the complex. Each element of the system or subsystem of entertainment complexes should be considered as A holistic system of A certain level, whic connects their components to the general purpose. Such systemic and structural principles will allow deeper understanding of this phenomenon and reliably determine the requirements and conditions for their formation and implementation at different hierarch cal levels, and can be effectively used for further research of trends and prospects for the development of modern leisure facilities' architecture. Further studies of this problem should also be directed to the study of the possibilities of using spaces and the formatio of the design of various existing buildings and struc tares, taking into account their con

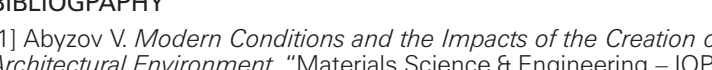

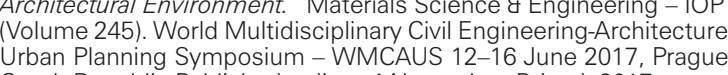
Czech Republic Published online: 4 November,
21 Eristol, 2017 . VISTKA, $2006 .-380 \mathrm{p}$.
3] Entertaining centers: yesterday, today, tomorrow ... Property Times No. 10.1351 .
[i] Petrova I.V. Entertaining complex in modern Ukraine: the prob-

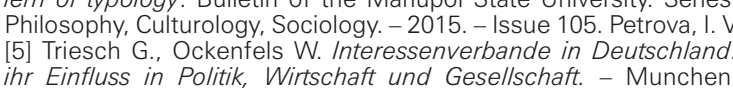

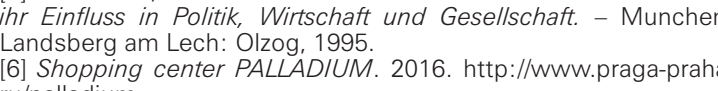

